1. Walba, D. M., Richards, R. M. \& Haltiwanger, R. C J. Am. Chem. Soc. 104, 3219-3221 (1982).

2. Mila, F., Stafford, C. \& Capponi, S. Phys. Rev. B 57, 1457-1460 (1998).

3. Hayashi, M. \& Ebisawa, H. J. Phys. Soc. Jpn 70, 3495-3498 (2001).

4. Kaneda, K. \& Okabe, Y. Phys. Rev. Lett. 86, 2134-2137 (2001).

5. Hearmon, R. F. S. An Introduction to Applied Anisotropic Elasticity (Oxford Univ. Press, London, 1961).

6. White, J. H. Am. J. Math. 91, 693-728 (1969).

7. Vologodskii, A. V., Anshelevich, V. V., Lukashin, A. V. \&

Endosymbiotic bacteria

\section{GroEL buffers against deleterious mutations}

roEL, a heat-shock protein that acts as a molecular chaperone ${ }^{1}$, is overproduced in endosymbiotic but not in free-living bacteria ${ }^{2-4}$, presumably to assist in the folding of conformationally damaged proteins. Here we show that the overproduction of GroEL in Escherichia coli masks the effects of harmful mutations that have accumulated during a simulated process of vertical transmission. This molecular mechanism, which may be an adaptation to the bacterium's intracellular lifestyle, is able to rescue lineages from a progressive fitness decline resulting from the fixation of deleterious mutations under strong genetic drift ${ }^{5,6}$.

Endosymbiotic bacteria have small population sizes, do not undergo recombination, and are maternally transmitted through tight population bottlenecks ${ }^{7}$, causing the fixation of deleterious mutations due to genetic drift and hence an irreversible decline in fitness ${ }^{8}$. However, endosymbiosis is surprisingly stable and persists over long periods ${ }^{9}$, which has led to the suggestion ${ }^{5}$ that groE (the GroEL-encoding operon) could be buffering the mutational loss of functionally active proteins because, unlike other endosymbiont genes, it is subject to strong purifying selection ${ }^{5}$.

To test this idea, we investigated the effects of overexpression of $g r o E$ in a set of E. coli strains (a free-living bacterium close to several endosymbionts ${ }^{9}$ ) with mutations randomly accumulated throughout the genome. These spontaneous mutations were fixed by random genetic drift in a process that simulated the vertical transmission of a single endosymbiont between hosts.

We studied the accumulation of mutations in 12 replicate lines of two E. coli B genotypes, one of which had a 3.3-foldincreased mutation rate ${ }^{10}$. These genotypes had already adapted to a simple environment (DM25 medium) for 10,000 generations $^{11}$, suggesting that mutation accumulation might result in a decline in fitness. After 3,240 generations of mutation accumulation, we measured the fitness of the strains evolved on DM25 relative to their respective ancestors. As expected, the
Frank-Kamenetskii, M. D. Nature 280, 294-298 (1979).

8. Tsutsumi, K. et al. Phys. Rev. Lett 39, 1675-1676 (1977)

9. Monceau, P. Electronic Properties of Inorganic Quasi-onedimensional Compounds (Reidel, Dordrecht, 1985).

10. Berry, M. V. Proc. R. Soc. Lond. A 392, 45-57 (1984).

11. Ando, T., Nakanishi, T. \& Saito, R. J. Phys. Soc. Jpn 67, 2857-2862 (1998).

12. Iijima, S. Nature 354, 56-58 (1991).

13. Hyde, S. T. Phys. Chem. Miner. 20, 190-200 (1993).

Competing financial interests: declared none.

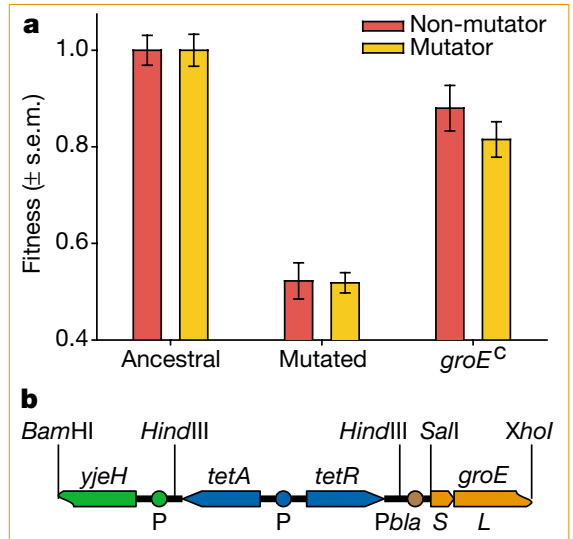

Figure 1 Effect of the overexpression of the groE operon on the fitness of randomly mutated strains of Escherichia coli. a, The fitness values for the ancestral strains of mutators and nonmutators are the same (left); 'mutated', fitness loss after 3,240 generations of mutation accumulation; ' $g r o E^{c}$ ', fitness recovery of strains in which groE is overexpressed. Culture conditions, fitness assays and phenotypic markers are described elsewhere ${ }^{11}$, as is the mutation-accumulation protocol ${ }^{12}$. b. The regulated $E$. coli groE operon was changed to a constitutive form ( $g r o E^{\mathrm{C}}$ ) by putting the structural genes under the control of the $\beta$-lactamase gene promoter (Pbla). Recombinant genotypes were obtained by transduction using P1 vir (ref. 13). The groE $E^{\mathrm{C}}$ construct contains part of the flanking $y j e H$ gene, the inducible tetracyclineresistance operon $($ tet $A / R)$ for transductant selection, the Pbla promoter, and the groE coding regions ( $\mathrm{S}$ and $\mathrm{L})$. Restrictionenzyme cutting sites are indicated; $\mathrm{P}$, promoter. Further details are available from the authors.

mutated strains lost fitness (Fig. 1a) as a result of the accumulation of deleterious mutations $(\bar{W}=0.5168 \pm 0.0159$ for mutators; $\bar{W}=0.5208 \pm 0.0087$ for non-mutators; paired $t$-tests, $P<0.0001$ ).

We then replaced the regulated groE operon of the mutated strains with a constitutive allele ( $g r o E^{c}$; Fig. 1b). To estimate the fitness of the $g r o E^{c}$ strains, we ran competition experiments against the corresponding ancestors on DM25. Surprisingly, none of the $g r o E^{c}$ strains reached the expected cell density during growth overnight. A simple explanation could be that an overproduction of GroEL of about $86 \pm 16$-fold is deleterious because it diverts amino acids away from other cellular functions.

To test this, we grew each $g r o E^{c}$ strain and its ancestor in DM25 supplemented with increasing concentrations of tryptone (a mixture of peptides and amino acids). We found that groE overexpression was deleteri- ous in the presence of small amounts of tryptone, but that there were no significant differences in cell density between $g r o E^{c}$ strains and their non-mutated ancestors at tryptone concentrations of $0.1 \%$ or higher. In endosymbionts, amino acids are not limiting because these are abundant in their intracellular environment ${ }^{9}$.

To determine whether fitness estimates depend on the environment, we estimated fitness on DM25 and on DM25+0.1\% tryptone. Both fitness estimates were correlated (partial correlation test, $P<0.0001$ ).

Figure 1a shows that the average fitness of the $g r o E^{\mathrm{c}}$ strains derived from nonmutator strains $(\bar{W}=0.8801 \pm 0.0214)$ was $75.9 \%$ greater than that of the mutated strains (paired $t$-test, $P<0.0001$ ), but was $12 \%$ less than that of the ancestors $(P=0.0002)$; the average fitness of the $g r o E^{c}$ strains derived from evolved mutators was $\bar{W}=0.8152 \pm 0.0167$, which is $61.6 \%$ greater than that of the mutated strains (paired $t$-test, $P<0.0001$ ) but $18.48 \%$ less than that of the ancestral strains $(P<0.0001)$.

Is fitness recovery a result of the buffering of deleterious effects by GroEL, or is it simply a general benefit associated with increased concentrations of GroEL? In favour of the first possibility, the advantage of GroEL overproduction is evident only in an amino-acid-rich environment; also, if mutation compensation is occurring, we would expect a positive correlation between the extents of fitness loss and recovery, as evidenced by their partial correlation (1-tailed test, $P=0.0089)$. We conclude that GroEL overexpression is likely to be of help in maintaining these endosymbionts by protecting them against the harmful effects of accumulated mutations.

Mario A. Fares, Mario X. Ruiz-González, Andrés Moya, Santiago F. Elena, Eladio Barrio

Institut Cavanilles de Biodiversitat i Biologia

Evolutiva and Departament de Genètica,

Universitat de València, PO Box 22085,

46071 València, Spain

e-mail:eladio.barrio@uv.es

1. Sigler, P. B. et al. Annu. Rev. Biochem. 67, 581-608 (1998).

2. Sato, S. \& Ishikawa, H. J. Bacteriol. 179, 2300-2304 (1997).

3. Aksoy, S. Insect Mol. Biol. 4, 32-29 (1995).

4. Charles, H., Heddi, A., Guillaud, J., Nardon, C. \& Nardon, P. Biochem. Biophys. Res. Commun. 239, 769-774 (1997).

5. Moran, N. A. Proc. Natl Acad. Sci. USA 93, 2873-2878 (1996).

6. Rutherford, S. L. \& Lindquist, S. Nature 396, 336-342 (1998).

7. Funk, D. J., Wernegreen, J. J. \& Moran, N. A. Genetics 157, 477-489 (2001).

8. Muller, H. J. Mutat. Res. 1, 2-9 (1964)

9. Moran, N. A. \& Wernegreen, J. J. Trends Ecol. Evol. 15, 321-326 (2000).

10. de Visser, J. A. G. M., Zeyl, C., Gerrish, P. J., Blanchard, J. L. \& Lenski, R. E. Science 283, 404-406 (1999).

11.Lenski, R. E., Rose, M. R., Simpson, S. C. \& Tadler, S. C. Am. Nat. 138, 1315-1341 (1991).

12. Kibota, T. T. \& Lynch, M. Nature 381, 694-696 (1996). 13. Zyskind, J. W. \& Bernstein, S. I. Recombinant DNA Laboratory Manual (Academic, San Diego, 1989).

Competing financial interests: declared none. 